\title{
A SELF-CONSISTENT TREATMENT OF THE $p-d$ MODEL BY MEANS OF THE COMPOSITE OPERATOR METHOD
}

\author{
F.MANCINI, S.MARRA, D.VILlani \\ INFM e Dipartimento di Fisica Teorica e S.M.S.A. \\ Universitá di Salerno , I-84081 Baronissi(SA), Italy
}

Received September 12, 1995

\begin{abstract}
A two-dimensional reduced p-d model describing $\mathrm{CuO}_{2}$ sheets in the high- $T_{c}$ oxides is studied by use of the composite operator approach. A set of self-consistent equations for the Green's function is derived in the static approximation for the paramagnetic state.
\end{abstract}

\section{Introduction}

The discovery of high- $T_{c}$ superconducting materials has revived the interest in the study of strongly correlated electron systems. This study requires theoretical methods completely different from those traditionally used in condensed matter physics. We have proposed a technique, denominated "composite operator method" (COM) [1], that has been applied to specific models like reduced $p-d$ model [2,3], Hubbard model [4], Heisenberg model [5]. Most of the theoretical scheme usually realize a perturbation approach in terms of the original fields. The COM is founded on the conviction that an analysis in terms of elementary fields might be inadequate for a system dominated by strong interactions. The original particle individuality disappears while the choice of new fundamental "particles", whose properties are determined in a self-consistent way by dynamics, symmetries and boundary conditions, becomes relevant. Due to the choice of a non-standard operatorial basis, COM generates some parameters not directly connected to the matrix elements for the Green's function. In our previous papers $[2,3]$, we treated those parameters as quantities given by hand to compute density of states and spectral densities or, in other words, as quantities that cannot be calculated within our approximation. The presence of such parameters, unlike foregoing point of view, does not result inconvenient because opens the opportunity to exceed the limits of our approximation. We have the possibility to vinculate the dynamics in a suitable Hilbert space, re absorbing the symmetries which control the structure and the coherence of the approximation. Definitely, an usual approximate theory selects a class of operatorially tractable diagrams which univocally determine a dynamics totally extraneous to the physical content of neglected diagrams; on the contrary, our approximation, denominated static approximation, even if realises a diagram choice, is capable to integrate the physical contribution of "lost" diagrams by means of constraining equations. The recover of the Pauli principle represents, in our new line of thought, a fundamental source 
of additional equations; the symmetry expressed by the exclusion principle can be recoverd by means of self-consistent determination of additional parameters.

Self-consistence and symmetries, which individuate the suitable Hilbert space and integrate the dynamics, represent the essence of the COM that appears, in conclusion, as a fully self-consistent technique for the study of systems in condensed matter physics.

The paper is organized as follows. In the next section the determination of reduced p-d Hamiltonian is summarized. The formula for the p-d model in COM framework is also presented. Some conclusion are given in Section 3 .

\section{The model}

Let us consider the highly correlated p-d model. Starting from the tightbinding model composed of $d$ electrons in $C u d_{x^{2}-y^{2}}$ orbitals and $p$ electrons in $\mathrm{O}_{p_{x}, p_{y}}$ on a $\mathrm{CuO}$ plane, taking only the transition to the upper Hubbard level (Cud $\Leftrightarrow C u d_{10}$ ), and considering only bonding $p$ electrons, we obtain the reduced p-d model $[2,6]$ described by the Hamiltonian:

$$
H=\sum_{i}\left\{\varepsilon_{p} p^{\dagger}(i) \cdot p(i)+\frac{1}{2} \varepsilon_{\eta} \eta^{\dagger}(i) \cdot \eta(i)+2 t\left[p_{\gamma}^{\dagger}(i) \cdot \eta(i)+\eta^{\dagger}(i) \cdot p_{\gamma}(i)\right]\right\},
$$

where $\varepsilon_{\eta}$ and $\varepsilon_{p}$ are bare energies of the upper Hubbard level and the $p$ electron level measured from the Fermi level, respectively, and

$$
\begin{aligned}
& \eta_{\sigma}(i)=d_{\sigma}(i) n_{-\sigma}(i), \\
& n_{\sigma}(i)=d_{\sigma}^{\dagger}(i) d_{\sigma}(i) .
\end{aligned}
$$

We use spinor notation:

$$
\begin{aligned}
p=\left(\begin{array}{c}
p_{\uparrow} \\
p_{\downarrow}
\end{array}\right), & p^{\dagger}=\left(p_{\uparrow}^{\dagger}, p_{\downarrow}^{\dagger}\right), \\
\eta=\left(\begin{array}{c}
\eta_{\uparrow} \\
\eta_{\downarrow}
\end{array}\right), & \eta^{\dagger}=\left(\eta_{\uparrow}^{\dagger}, \eta_{\downarrow}^{\dagger}\right) .
\end{aligned}
$$

The $t$ term describes the $p-\eta$ hopping, with $p_{\gamma}(i)$ defined by

$$
p_{\gamma}(i)=\sum_{j} \gamma_{i j} p(j)=\frac{\Omega}{(2 \pi)^{2}} \int_{\Omega_{B}} d^{2} k e^{i \mathbf{k} \cdot \mathbf{R}_{i}} \gamma(\mathbf{k}) p(\mathbf{k})
$$

with

$$
\gamma(\mathbf{k})=\sqrt{1-\alpha(\mathbf{k})}
$$

where for a two-dimensional quadratic lattice with lattice constant $a$

$$
\alpha(\mathbf{k})=\frac{1}{2}\left[\cos \left(k_{x} a\right)+\cos \left(k_{y} a\right)\right] .
$$

Since the transition to the upper Hubbard level corresponds to the $(n=$ $1) \Leftrightarrow(n=2)$ transitions of the $d$ electron number, Hamiltonian in (2.1) is supplemented by the condition $(n-1)(n-2)=0$. 
The doping dependence of the electronic state is not rigid-band-like, but an intensity transfer from the high-energy region to the low-energy region is induced [7]. When holes are doped in the system, the mixing induces a non-linear many body effect accompanied by spin fluctuations and creates a new narrow band around the Fermi level inside the charge transfer energy gap. The new band develops to a wide metallic one with hole doping. These effects cannot be reached, in a simple way, by using atomic degrees of freedom; on the contrary, by means of the COM, we treat the developed state as a stable excitation or, more precisely, as a fundamental field. In the $p-d$ model of oxide superconductors, we expect the excitations to be primarily local, since the system is close to a ionic crystal. Then, we select as fundamental fields the oxygen $p$ - electron state, the copper $d$ electron operator constrained to low-energy sector, the $p$-electron excitation accompanying the copper spin fluctuations and the $p$-electron excitation accompanying the copper charge fluctuations.

Therefore, we introduce the field operator

$$
\psi(i)=\left(\begin{array}{c}
p(i) \\
\eta(i) \\
p_{s}(i) \\
p_{0}(i)
\end{array}\right)
$$

where

$$
\begin{aligned}
& p_{s}(i)=\sigma_{k} n_{k}(i) p_{\gamma}(i) \\
& p_{0}(i)=n(i) p_{\gamma}(i)
\end{aligned}
$$

with $n_{k}(i)=d_{\sigma}^{\dagger}(i)\left(\sigma_{k}\right)_{\sigma \delta} d_{\delta}(i)$ and $n(i)=d^{\dagger}(i) \cdot d(i) . \sigma_{k}(k=1,2,3)$ are the Pauli matrices. The operator $p_{s}(i)$ represents the $p$-electron excitation accompanying the $C u$ spin fluctuation whereas $p_{0}(i)$ represents the $p$-electron excitation accompanying the $C u$ charge fluctuation.

The introduction of a new composite operator does not mean to add a new operator freedom in the formalism but corresponds to a procedure of choosing a suitable asymptotic field, in the field theoretical language, for a new bound state which appears in addition to fundamental particle state. The Heisenberg operator $\psi(i)$ satisfies the equation of motion

$$
i \frac{\partial}{\partial t} \psi(i)=j(i)
$$

The source term $j(i)$ is computed by means of the Hamiltonian (2.1) and has the expression

$$
j(i)=\left(\begin{array}{c}
\varepsilon_{p} p(i)+2 t \eta_{\gamma}(i) \\
\varepsilon_{\eta} \eta(i)-t p_{s}(i)+t p_{0}(i) \\
\varepsilon_{p} p_{s}(i)+2 t \kappa_{s} \\
\varepsilon_{p} p_{0}(i)+2 t \kappa_{0}
\end{array}\right),
$$

with

$$
\begin{aligned}
& \kappa_{s}(i)=\sigma_{k} \eta^{\dagger}(i) \sigma_{k} p_{\gamma}(i) p_{\gamma}(i)-\sigma_{k} p_{\gamma}^{\dagger}(i) \sigma_{k} \eta(i) p_{\gamma}(i)+\sigma_{k} n_{k} \eta_{\gamma^{2}}(i) \\
& \kappa_{0}(i)=\eta^{\dagger}(i) p_{\gamma}(i) p_{\gamma}(i)-p_{\gamma}^{\dagger}(i) \eta(i) p_{\gamma}(i)+n(i) \eta_{\gamma^{2}}(i)
\end{aligned}
$$


To describe the properties of the system we introduce the two-point retarded thermal Green's function:

$$
S(i, j)=\left\langle R\left[\psi(i) \psi^{\dagger}(j)\right]\right\rangle,
$$

where the bracket indicates the thermal average and $R$ is the usual retarded operator. When we want to use the equation of motion to compute the Green's functions, a convenient technique is to decompose $j(i)$ under the form:

$$
j(i)=\epsilon(-i \vec{\nabla})+\delta j(i),
$$

with the constrain:

$$
\left\langle\left\{\delta j_{n}(i), \psi_{m}^{\dagger}(j)\right\}\right\rangle_{E . T .}=0 .
$$

This technique, first introduced in nuclear physics [8], has been adopted by many authors [9]. By standard calculations, the Fourier transform $S(\mathbf{k}, \omega)$ can be computed by means of the Heisenberg equation and takes the expression :

$$
S(\mathbf{k}, \omega)=\frac{1}{\omega-\epsilon(\mathbf{k})-\Sigma(\mathbf{k}, \omega)} I(\mathbf{k}),
$$

where the kinetic $\varepsilon(\mathbf{k})$ and the dynamical $\Sigma(\mathbf{k}, \omega)$ parts of the self energy are given by

$$
\begin{aligned}
\epsilon(\mathbf{k}) & =m(\mathbf{k}) I^{-1}(\mathbf{k}) \\
\Sigma(\mathbf{k}, \omega) & =M(\mathbf{k}, \omega) S^{-1}(\mathbf{k}, \omega)
\end{aligned}
$$

with

$$
\begin{aligned}
I(\mathbf{k}) & =\left\langle\left\{\psi(i), \psi^{\dagger}(j)\right\}\right\rangle_{F . T}, \\
m(\mathbf{k}) & =\left\langle\left\{j(i), \psi^{\dagger}(j)\right\}\right\rangle_{F . T}, \\
M(\mathbf{k}, \omega) & =\left\langle R\left[\delta j(i), \psi^{\dagger}(j)\right]\right\rangle_{F . T} .
\end{aligned}
$$

equation (2.19) is only a formal solution; in order to explicitly calculate the Green's function we must introduce some approximation. In our previous works we have computed $S(\mathbf{k}, \omega)$ by neglecting the dynamical part $\Sigma(\mathbf{k}, \omega)$ in the self energy (in this sense we might indicate this approximation as "static approximation") reproducing with quite good accuracy many of the results obtained by means of exact numerical calculations on finite size systems. Therefore, we need the knowledge of the normalization matrix given by (2.22) and of the $m$-matrix, defined by (2.23). As boundary condition, we assume a paramagnetic ground state

$$
\left\langle n_{\uparrow}(i)\right\rangle=\left\langle n_{\downarrow}(i)\right\rangle=n / 2 .
$$

A straightforward calculation gives

$$
\begin{aligned}
I(\mathbf{k}) & =\left(\begin{array}{cccc}
1 & 0 & 0 & \gamma(\mathbf{k}) n \\
0 & n / 2 & 3 b & b \\
0 & 3 b & I_{33} & 0 \\
\gamma(\mathbf{k}) n & b & 0 & I_{44}
\end{array}\right), \\
m(\mathbf{k}) & =\left(\begin{array}{cccc}
\varepsilon_{p} & n t \gamma(\mathbf{k}) & 6 t \gamma(\mathbf{k}) b & \left(\varepsilon_{p} n+2 t b\right) \gamma(\mathbf{k}) \\
n t \gamma(\mathbf{k}) & (n / 2) \varepsilon_{\eta}-2 t b & m_{23} & m_{24} \\
6 t \gamma(\mathbf{k}) b & m_{32} & m_{33} & m_{34} \\
\left(\varepsilon_{p} n+2 t b\right) \gamma(\mathbf{k}) & m_{42} & m_{43} & m_{44}
\end{array}\right)
\end{aligned}
$$


where

$$
\begin{aligned}
I_{33} & =4 a_{s}+3 \chi_{s}-3 \chi_{s}^{\prime} \alpha(\mathbf{k}), \\
I_{44} & =\chi_{0}-\chi_{0}^{\prime} \alpha(\mathbf{k}), \\
m_{23} & =m_{32}=3 \varepsilon_{\eta} b-t I_{33}, \\
m_{24} & =m_{42}=\varepsilon_{\eta} b+t I_{44} \\
m_{33} & =\varepsilon_{p} I_{33}-4 t\left[3 b+2 b_{s}^{\prime}-3 d_{s} \alpha(\mathbf{k})+3 f\right], \\
m_{34} & =m_{43}=6 t\left[2 b-\left(d_{0}+d_{s}\right) \alpha(\mathbf{k})+2 f\right], \\
m_{44} & =\varepsilon_{p} I_{44}+4 t\left[b-d_{0} \alpha(\mathbf{k})-f\right] .
\end{aligned}
$$

We are using the following notation:

$$
\begin{aligned}
n & =\left\langle d^{\dagger}(i) d(i)\right\rangle \\
b & =\left\langle\eta(i) p_{\gamma}^{\dagger}(i)\right\rangle \\
a_{s} & =\left\langle p_{\gamma}(i) p_{s}^{\dagger}(i)\right\rangle \\
\chi_{s} & =\frac{1}{3}\left\langle n_{k}(i) n_{k}(i)\right\rangle=2\left\langle\eta(i) \eta^{\dagger}(i)\right\rangle=2-n \\
\chi_{s}^{\prime} & =\frac{1}{3}\left\langle n_{k}(i) n_{k}^{\alpha}(i)\right\rangle \\
\chi_{0} & =\langle n(i) n(i)\rangle=3 n-2 \\
\chi_{0}^{\prime} & =\left\langle n(i) n^{\alpha}(i)\right\rangle \\
b_{s}^{\prime} & =\left\langle\eta^{\alpha}(i) p_{s}^{\dagger}(i)\right\rangle \\
d_{s} & =\frac{1}{3}\left\langle\sigma_{k} n_{k}^{\alpha}(i) \eta(i) p_{\gamma}^{\dagger}(i)\right\rangle \\
d_{0} & =\left\langle n^{\alpha}(i) \eta(i) p_{\gamma}^{\dagger}(i)\right\rangle \\
f & =\left\langle p_{\gamma}(i) \eta^{\dagger}(i) p_{\gamma}(i) p_{\gamma}^{\dagger}(i)\right\rangle .
\end{aligned}
$$

The quantities $n, b, a_{s}, \chi_{s}, \chi_{0}$ and $b_{s}^{\prime}$ are self-consistent parameters in the sense that can be expressed in terms of matrix elements related to the fermion propagator. The parameters $\chi_{s}^{\prime}, \chi_{0}^{\prime}, d_{s}, d_{0}$ and $f$ are not directly expressed in terms of the one-particle Green's function. In the COM we take advantage of this internal freedom in order to construct the Hilbert space with the right symmetries. In fact, by means of the following sum rules

$$
\begin{aligned}
\left\langle p_{s}(i) \eta^{\dagger}(i)\right\rangle & =0 \\
\left\langle p_{0}(i) \eta^{\dagger}(i)\right\rangle & =2 b \\
\left\langle p_{s}(i) p_{s}^{\dagger}(i)\right\rangle & =6\left\langle p_{\gamma}(i) p_{\gamma}^{\dagger}(i)\right\rangle-3\left\langle p_{\gamma}(i) p_{0}^{\dagger}(i)\right\rangle+2\left\langle p_{\gamma}(i) p_{s}^{\dagger}(i)\right\rangle \\
\left\langle p_{s}(i) p_{0}^{\dagger}(i)\right\rangle & =\left\langle p_{s}(i) p_{\gamma}^{\dagger}(i)\right\rangle \\
\left\langle p_{0}(i) p_{0}^{\dagger}(i)\right\rangle & =3\left\langle p_{0}(i) p_{\gamma}^{\dagger}(i)\right\rangle-2\left\langle p_{\gamma}(i) p_{\gamma}^{\dagger}(i)\right\rangle
\end{aligned}
$$

we can recover the content of the Pauli principle that controls the algebra of our non-standard operatorial basis. 
The Fermi level is fixed by the self-consistent equation:

$$
4-2\left(\left\langle p(i) p^{\dagger}(i)\right\rangle+\left\langle\eta(i) \eta^{\dagger}(i)\right\rangle\right)=\left\langle n_{T}\right\rangle,
$$

where $\left\langle n_{T}\right\rangle$ is the total number of particles per unit $\mathrm{CuO}$ cell. Then, there are no adjustable parameters and the formulation is fully self-consistent. The solution of the set of self-consistent equations (2.46)-(2.51) allow us to compute the fermion Green's function.

\title{
3. Conclusions
}

In the static approximation, we have obtained a fully self-consistent solution for the reduced p-d model. A set of sum rules, with content of Pauli principle, discloses the possibility to realize a self-consistent theory and to recover important coherent contributions of diagrams usually neglected in a ordinary mean-field theory. Numerical solutions of these equations and physical properties of the system will be presented elsewhere.

\section{References}

[1] Matsumoto H., Sasaki M., Ishihara I., Tachiki M. Composite-operator approach for the $p-d$ mixing model of oxide superconductors. // Phys. Rev. B, 1992, vol. 46, p. 3009-3021;

Sasaki M., Matsumoto H., Tachiki M. Electronic states of high- $T_{c}$ cuprate superconductors as studied by the use of the composite-operator approach. // Phys. Rev. B, 1992, vol. 46, p. 3022-3040.

[2] Ishihara S., Matsumoto H., Odashima S., Tachiki M., Mancini F. Mean-field analysis in the $p-d$ model of oxide superconductors. // Phys. Rev. B, 1994, vol. 49 , p. $1350-1365$.

[3] Mancini F., Marra S., Villani D., Matsumoto H. Local magnetic moment in the reduced $p-d$ model. // Phys. Lett. A, 1996.

[4] Mancini F., Marra S., Matsumoto H. Doping dependence of on-site quantities in the two-dimensional Hubbard model. // Physica C, 1995, vol. 244, p. 49-53; Mancini F., Marra S., Matsumoto H. Energy and chemical potential in the two-dimensional Hubbard model. // Physica C, 1995, vol. 250, p. 184-190.

[5] Allega A.M., Odashima S., Matsumoto H., Mancini F. Static and dynamical spin susceptibility in 2D antiferromagnetic Heisenberg model. // Physica C, 1994, vol. 235-240, p. 2229-2230.

[6] Matsumoto H., Tachiki M. Metal-insulator transition in oxide superconductors. // Progr. Theor. Phys. Supplement, 1990, vol. 101, p. 353-369.

[7] Ohta Y., Tsutsui K., Koshibae W., Shimozato T., Maekawa S. Evolution of the in-gap state in high- $T_{c}$ cuprates. // Phys. Rev. B, 1992, vol. 46, p. 14022-14033.

[8] Dancoff S.M. Non-adiabatic meson theory of nuclear forces. // Phys. Rev., 1950, vol. 78 , p. 382-390;

Rowe D.J. Equations-of-motion method and the extended shell model. // Rev. Mod. Phys., 1968, vol. 40, p. 153-166.

[9] Roth L.M. Electron correlation in narrow energy bands. I. The two-pole approximation in a narrow S band. // Phys. Rev., 1969, vol. 184, p. 451-459.

\section{САМОУЗГОДЖЕНИЙ ПІДХІД У $p-d$ МОДЕЛІ МЕТОДОМ КОМПОЗИТНОГО ОПЕРАТОРА}

\author{
Ф.Манчіні, С.Марра, Д.Віллані
}

Двовимірна спрощена $p-d$ модель, що описує шари $\mathrm{CuO}_{2}$ в ВТНП оксидах досліджується з використанням методу композитного оператора. Отримується самоузгоджений набір рівнянь для функцій Гріна в статистичному наближенні по парамагнітному стану. 